\title{
Optical positions and proper motions of radio stars
}

\author{
P.Z. Lu and T.Q. Xu \\ Shanghai Observatory, Academia Sinica, 200030 Shanghai, China
}

Received July 30; accepted August 28, 1996

\begin{abstract}
The optical positions of 31 radio stars were obtained with the $40 \mathrm{~cm}$ astrograph at Zô-Sè Section, Shanghai Observatory from 1985 to 1988 . The observational positions and the proper motions of these radio stars in the FK5 system are given in this paper.
\end{abstract}

Key words: astrometry — radio continuum: stars — reference systems

\section{Introduction}

From 1985 to 1988 , the $40 \mathrm{~cm}$ astrograph $(f=6.9 \mathrm{~m})$ at Zô-Sè Section, Shanghai Observatory was used to observe the radio stars (Xu et al. 1990). The radio stars were selected by (1) the Working Group on the identification of the radio/optical astrometric sources of the IAU Commission 24 and (2) the Hipparcos Input List of the radio stars. The reference catalogues used were the AGK3 for the observations in the northern hemisphere and the SAOC for the observations in the southern hemisphere, respectively. Now these observational results have been reprocessed in the FK5 system.

Among these there are 31 radio stars found in the AGK3 and SAOC catalogues. The proper motions of these radio stars were obtained from a comparison between the positions observed and that of the AGK3, SAOC catalogues.

The results have also been compared with those given in the PPM and CAMC catalogues.

\section{Reductions of the optical positions and the proper motions}

The reductions of the observational positions were performed as described in $\mathrm{Xu}$ et al. (1990). In the process, the different systematic differences (FK5-reference catalogues) were added to the positions of the reference catalogues with the aim of reducing the reference catalogues used in the FK5 system. The systematic differences (FK5-AGK3/SAOC) were taken using the method of
Bien et al. (1978). The results were given in detail by $\mathrm{Lu}$ et al. (1996a).

The reduction procedure of the proper motions was as same as described in Lu et al. (1996b).

\section{Results}

The observational positions and proper motions for 31 radio stars are presented in Table 1: in Col. 1 is the serial number, in Col. 2 the name of the radio stsrs, in Col. 3 the magnitude, in Cols. 4 and 6 are the right ascension and declination for the observational epoch and equinox J2000.0, in Cols. 5 and 7 are the mean square error in right ascension and in declination, respectively, in Cols. 8 and 9 are the proper motion per year in right ascension and its mean square error, in Cols. 10 and 11 are the proper motion per year in declination and its mean square error, in Col. 12 is the mean epoch of observation and in Col. 13 is the serial number of the Hipparcos Input Catalogue.

Some of these radio stars are also found in the PPM (Röser et al. 1989) and CAMC (CAMC 1989) catalogues. In Figs. $1 \mathrm{a}$ and $1 \mathrm{~b}$ the differences of the proper motions both (Shanghai-PPM) and (Shanghai-CAMC) in right ascension and declination are shown respectively. In Figs. $2 \mathrm{a}$ and $2 \mathrm{~b}$ the differences of the proper motions respectively between PPM and CAMC in right ascension and declination are given. The comparison between Figs. 1 and 2 indicates that our results of the proper motions coincide in general with those of the PPM and CAMC catalogues. But for the proper motion in right ascension of the radio star $\mathrm{R} C O M$, there is possibly a few deviations between the PPM and the CAMC or ours.

Acknowledgements. The authors wish to thank Dr. H. Schwan, Astronomisches Rechen-Institut Heidelberg, for his valuable discussion in the process of the catalogue differences. This work is supported by the National Natural Science Foundation of China (No. 19073014). 
Table 1. Observational positions and proper motions of 31 radio stars

\begin{tabular}{|c|c|c|c|c|c|c|c|c|c|c|c|c|c|c|c|c|}
\hline No & Name & $\begin{array}{r}\mathrm{Mag} \\
(\mathrm{m})\end{array}$ & & $\begin{array}{r}R . \\
(\mathbf{J} 200\end{array}$ & & $\begin{array}{r}\text { S.A } \\
\text { (ms) }\end{array}$ & & $\begin{array}{l}\mathrm{DE} \\
200 \\
\end{array}$ & & $\begin{array}{r}\text { S.D } \\
\left(0^{\prime \prime} .01\right) \\
\end{array}$ & $\begin{array}{r}\text { PMA } \\
(0.1 \mathrm{~ms}) \\
\end{array}$ & $\begin{array}{r}\text { SPMA } \\
(0.1 \mathrm{~ms}) \\
\end{array}$ & $\begin{array}{r}\text { PMD } \\
(0 " .001) \\
\end{array}$ & $\begin{array}{r}\text { SPMD } \\
(0 " .001) \\
\end{array}$ & $\begin{array}{r}\text { Epoch } \\
(1900+) \\
\end{array}$ & Hip \\
\hline & & & h & $\mathbf{m}$ & $\mathrm{s}$ & & 0 & & 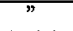 & & & & & & & \\
\hline 1 & UV PSC & 9.1 & 01 & 16 & 55.034 & 6 & 06 & 48 & 41.79 & 11 & 57 & 2 & 18 & 3 & 86.91 & 5980 \\
\hline 2 & O CET & 7.3 & 02 & 19 & 20.796 & 13 & -02 & 58 & 36.76 & 9 & -1 & 2 & -243 & 2 & 86.78 & $10826 \mathrm{AP}$ \\
\hline 3 & S PER & 10.6 & 02 & 22 & 51.682 & 4 & 58 & 35 & 11.40 & 8 & -3 & 3 & -1 & 4 & 86.32 & $11093 \mathrm{~A}$ \\
\hline 4 & YY ERI & 8.5 & 04 & 12 & 08.917 & 26 & -10 & 28 & 08.82 & 22 & -82 & 4 & -120 & 5 & 86.93 & 19610 \\
\hline 5 & T TAU & 10.8 & 04 & 21 & 59.415 & 5 & 19 & 32 & 06.18 & 8 & 7 & 2 & -16 & 3 & 86.03 & 20390 \\
\hline 6 & RU CNC & 10.3 & 08 & 37 & 30.136 & 4 & 23 & 33 & 41.46 & 10 & -14 & 2 & -1 & 3 & 86.03 & 42303 \\
\hline 7 & RL MIN & 10.8 & 09 & 45 & 34.281 & 11 & 34 & 30 & 42.77 & 10 & 2 & 3 & -3 & 2 & 86.10 & 47886 \\
\hline 8 & AD LEO & 10.0 & 10 & 19 & 36.773 & 6 & 19 & 52 & 12.23 & 11 & -350 & 2 & -48 & 3 & 86.18 & \\
\hline 9 & R CRT & 9.2 & 11 & 00 & 33.974 & 8 & -18 & 19 & 29.51 & 14 & -18 & 2 & 2 & 3 & 87.10 & $53809 \mathrm{~A}$ \\
\hline 10 & R COM & 12.1 & 12 & 04 & 15.177 & 11 & 18 & 46 & 55.96 & 7 & -8 & 2 & -11 & 3 & 88.36 & 58854 \\
\hline 11 & RT VIR & 8.3 & 13 & 02 & 37.960 & 7 & 05 & 11 & 08.65 & 13 & 26 & 2 & -7 & 3 & 86.42 & 63642 \\
\hline 12 & RS CVN & 8.1 & 13 & 10 & 36.959 & 8 & 35 & 56 & 04.91 & 18 & -35 & 2 & 10 & 4 & 87.10 & 64293 \\
\hline 13 & V CVN & 7.9 & 13 & 19 & 27.818 & 7 & 45 & 31 & 37.87 & 5 & -32 & 2 & -11 & 3 & 88.29 & 65006 \\
\hline 14 & FK COM & 8.2 & 13 & 30 & 46.852 & 11 & 24 & 13 & 57.90 & 7 & -37 & 2 & -19 & 3 & 88.32 & $65915 \mathrm{C}$ \\
\hline 15 & S VIR & 10.8 & 13 & 33 & 00.116 & 9 & -07 & 11 & 41.28 & 21 & -20 & 2 & 1 & 3 & 86.81 & 66100 \\
\hline 16 & ZZ BOO & 6.9 & 13 & 56 & 09.617 & 4 & 25 & 55 & 07.11 & 16 & -66 & 3 & -6 & 4 & 88.28 & 68064 \\
\hline 17 & RV LIB & 9.1 & 14 & 35 & 48.493 & 22 & -18 & 02 & 11.35 & 15 & -9 & 4 & -15 & 4 & 88.30 & 71380 \\
\hline 18 & S CRB & 11.1 & 15 & 21 & 23.961 & 8 & 31 & 22 & 02.43 & 17 & -6 & 3 & -20 & 4 & 86.43 & 75143 \\
\hline 19 & S SER & 11.9 & 15 & 21 & 39.514 & 7 & 14 & 18 & 52.84 & 7 & -5 & 2 & 5 & 3 & 88.30 & 75170 \\
\hline 20 & R SER & 11.2 & 15 & 50 & 41.702 & 6 & 15 & 08 & 02.28 & 12 & -1 & 2 & -32 & 3 & 88.52 & 77615 \\
\hline 21 & U HER & 11.0 & 16 & 25 & 47.478 & 12 & 18 & 53 & 32.87 & 13 & -4 & 3 & -6 & 4 & 86.43 & 80488 \\
\hline 22 & RY SCT & 9.4 & 18 & 25 & 31.517 & 12 & -12 & 41 & 23.63 & 15 & 6 & 3 & 1 & 4 & 85.58 & 90303 \\
\hline 23 & R AQL & 9.7 & 19 & 06 & 22.249 & 4 & 08 & 13 & 48.67 & 5 & 5 & 2 & -65 & 2 & 87.72 & $93820 \mathrm{~A}$ \\
\hline 24 & CYG X-1 & 8.8 & 19 & 58 & 21.681 & 6 & 35 & 12 & 06.02 & 14 & 1 & 2 & -2 & 3 & 85.58 & 98298 \\
\hline 25 & CYG OB2-5 & 9.1 & 20 & 32 & 22.421 & 6 & 41 & 18 & 18.89 & 6 & -2 & 2 & -7 & 3 & 85.63 & 101341AB \\
\hline 26 & RT LAC & 9.4 & 22 & 01 & 30.681 & 6 & 43 & 53 & 25.19 & 9 & 52 & 3 & 21 & 5 & 85.64 & 108728 \\
\hline 27 & TW PEG & 6.6 & 22 & 03 & 59.516 & 4 & 28 & 20 & $\mathbf{5 4 . 3 5}$ & 6 & 12 & 2 & -4 & 3 & 85.78 & 108928 \\
\hline 28 & DM-21 6267 & 9.1 & 22 & 38 & 45.130 & 19 & -20 & 37 & 15.04 & 24 & 319 & 6 & -95 & 7 & 86.91 & $111802 \mathrm{~A}$ \\
\hline 29 & $D M+542846$ & 8.8 & 22 & 44 & 07.469 & 8 & 55 & 35 & 21.74 & 12 & 9 & 2 & 5 & 3 & 86.77 & 112247 \\
\hline 30 & EV LAC & 10.3 & 22 & 46 & 51.527 & 9 & 44 & 20 & 22.07 & 10 & 4 & 2 & -4 & 2 & 86.68 & $112460 \mathrm{~A}$ \\
\hline 31 & R PEG & 11.4 & 23 & 06 & 39.173 & 5 & 10 & 32. & 36.29 & 8 & 8 & 2 & -11 & 2 & 87.71 & 114114 \\
\hline
\end{tabular}
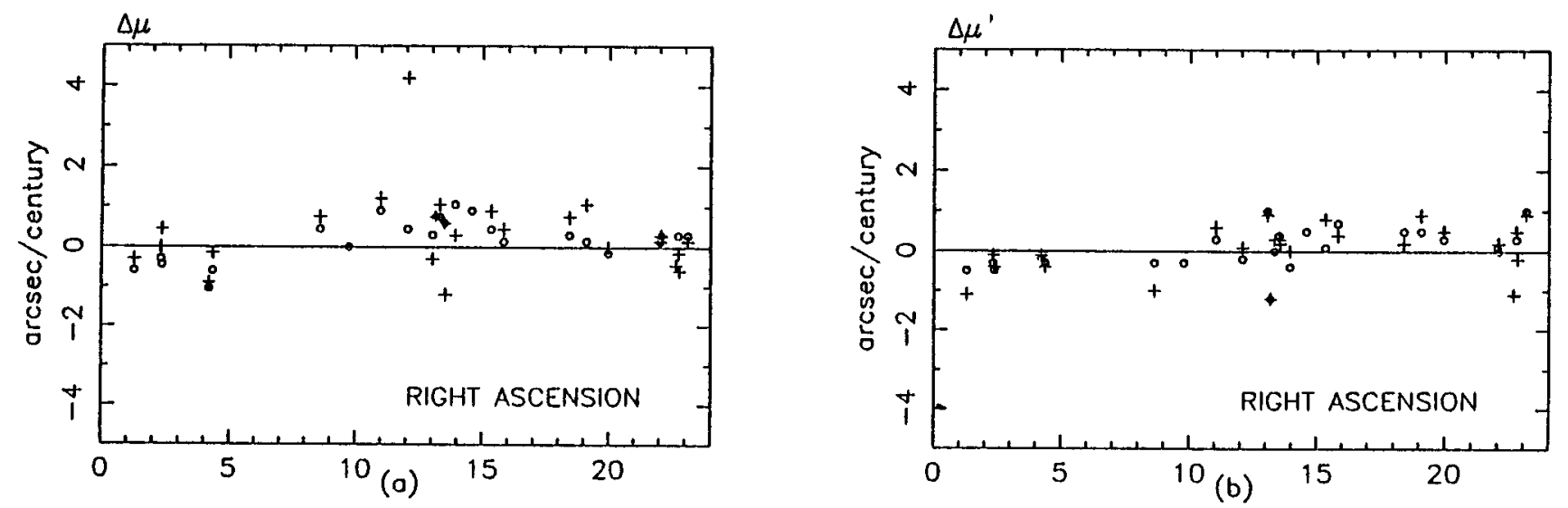

Fig. 1. Differences of the proper motions in right ascension a) and declination; b) $\circ($ Shanghai-CAMC); +: (Shanghai-PPM)

\section{References}

Bien R., Fricke W., Lederle T., Schwan H., 1978, Veröffentlichungen ARI Heidelberg, No. 29

Carlsberg Meridian Catalogue La Palma, No. 4, 1989, Copenh. Univ. Obs., R. Greenwich Obs., R. Inst. Obs. Armada en San Fernando
Lu P.Z., Xu T.Q., 1996a, Ann. Shanghai Obs. Acad. Sin. 17 Lu P.Z., Xu T.Q., 1996b, A\&AS 115, 311

Röser S., Rastian U., 1989, PPM star Catalogue, ARI Heidelberg

Xu T.Q., Lu P.Z., Wang S.H., Chu Z.Y., 1990, Acta Astron. Sin. 31,267 

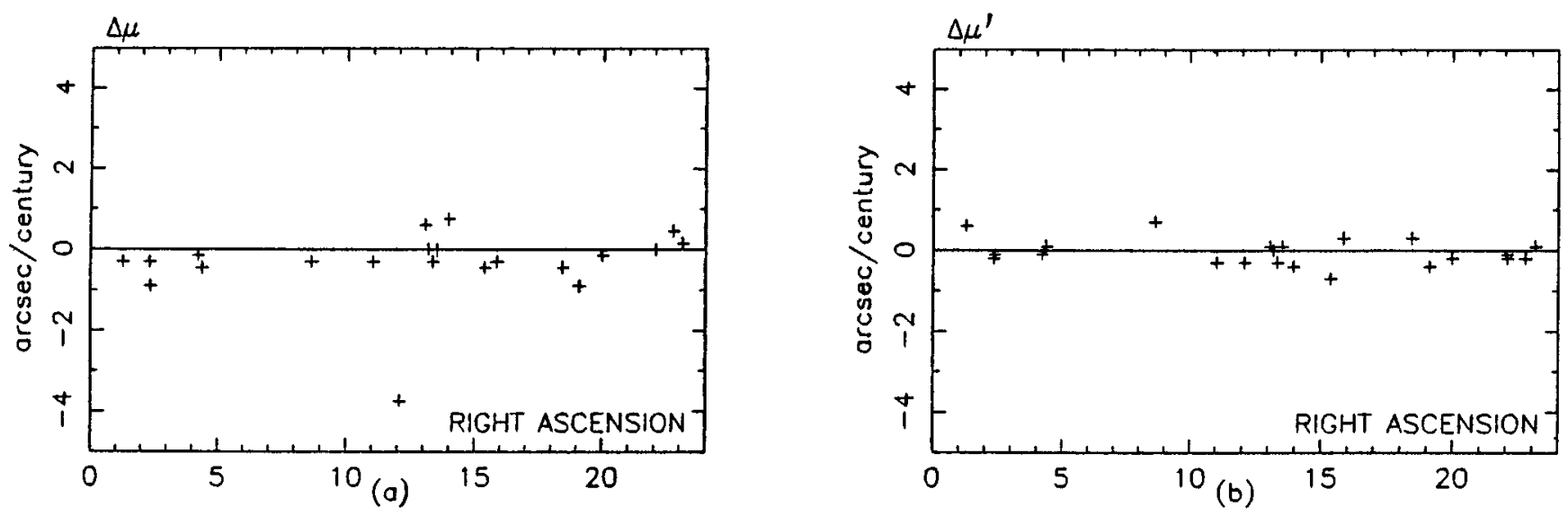

Fig. 2. Differences of the proper motions in right ascension a) and declination; b) (PPM-CAMA) 